\section{§23. Automatic Tracking of Low-Pressure Vortex}

\author{
Makihara, T., Kida, S., Miura, H.
}

Tubular swirling vortices are commonly observed in many kinds of turbulent flows. They play central roles in turbulence dynamics. This suggests that understanding of their dynamical properties should be a prerequisite for the prediction and control of turbulence.

The individual vortices, if identified, may have their own lives of finite length of time. It is anticipated that they are created through a kind of Kelvin-Helmholtz instability, interact with other vortices as well as with the background shear flow, and then break down into scattered vorticity, which becomes the source of new vortices, and so on. Although plausible, this scenario is hard to observe because a lot of other vortices are moving randomly in a turbulent flow, which may hide this typical scenario. It would therefore be helpful if an arbitrarily chosen vortex could be tracked automatically.

Tracking of an individual vortex is not an easy task because they are not conserved objects. They may split, merge, disappear, or even be newly created. Nevertheless, we develop here an automatic tracking scheme for vortex axes. The vortex tracking is carried out by identifying vortices at different times. At each time instant, a vortex is extracted as a low-pressure vortex which is a rotating low-pressure region ${ }^{1}$.

The identification scheme for vortex axes at two consecutive times is constructed based upon the idea of 'majority decision'. Vortices that take many votes both from the master grid points of all the nodes on a chosen vortex axis at a previous time and from their neighbors are identified as descendants of the vortex of interest. The numerical procedure for this automatic tracking scheme is composed of the following six steps (see Fig. 1).

[1] Prepare two data sets of vortex axes at times $t$ and $t+\Delta t$, where $\Delta t$, being larger than the time step width employed in the numerical simulation, is set to be sufficiently small that the vortices may move at most by the grid width $\Delta x$.

[2] Choose a vortex axis to be tracked at time $t$.

[3] Pick up all the master grid points associated with this vortex (denoted by solid circles in Fig. 1).

[4] Register the above master grid points and their neighbors (open circles) as voters.

[5] Each of these registered grid points gives a vote to the vortex axis to which it belongs at time $t+\Delta t$.

[6] Count the number of votes all the vortex axes received at time $t+\Delta t$. Descendants of the original vortex are determined by the following two criteria. First, the vortex axes that receive only a single vote or that have node points more than double the original one are discarded. Then, those that have the largest votes or receive more than $n_{c}(=8 \sim 10$, say $)$ votes are identified as descen-

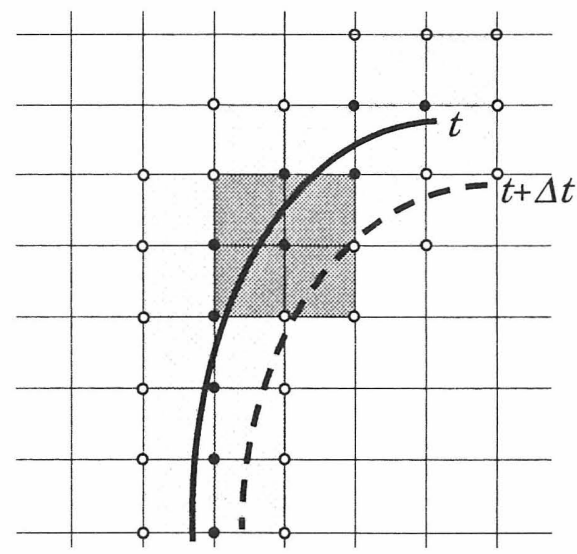

Fig. 1 Two-dimensional illustration of tracking scheme

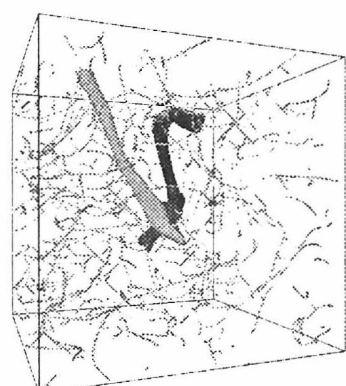

(a) $t=0$

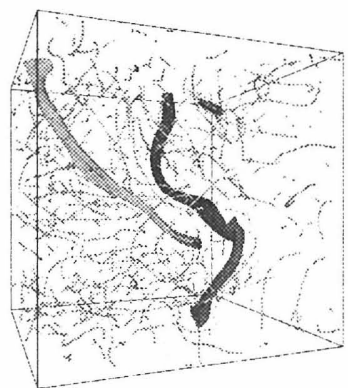

(c) $t=4.71 \tau_{K}$ (b) $t=2.36 \tau_{K}$

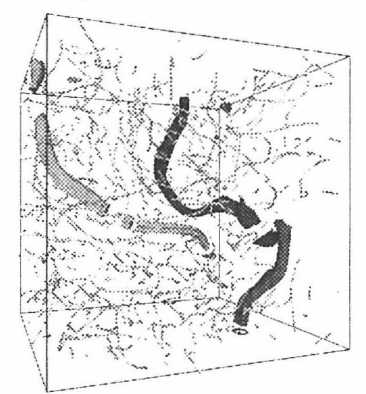

(d) $t=7.07 \tau_{K}$

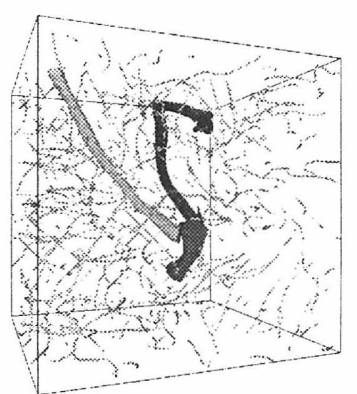

Fig. 2 Tracking of low-pressure vortices

dants.

This tracking scheme is applied to a forced turbulence of the Reynolds number $R_{\lambda}=85$ at a resolution of $256^{3}$ over a period of $7.07 \tau_{K}$, where $\tau_{K}$ is the Kolmogorov time. The results are shown in Fig. 2, where the cores of three arbitrarily chosen vortices are represented in a cubic region of side length $128 \Delta x$. The trackings are repeated 13 times between consecutive panels. All other vortex axes are represented with thin lines. By this method we can easily track any vortex to examine its entire life, from generation to break-down.

\section{Reference}

1)S. Kida and H. Miura, Eur. J. Mech. B/Fluids 17 (1998) 471. 